\title{
Techniques and outcomes of bronchoplastic and sleeve resection: an 8-year single-center experience
}

\author{
Xin-Yu Zhu ${ }^{1,2 \#}$, Cheng Ding ${ }^{1,2 \#}$, Chun $\mathrm{Xu}^{1,2}$, Jun Chen ${ }^{1,2}$, Sheng Ju ${ }^{1,2}$, Shu Pan ${ }^{1,2}$, Zi-Han Cui ${ }^{1,2}$, \\ Benoît Bédat ${ }^{3}$, Duilio Divisi ${ }^{4}$, Davide Tosi ${ }^{5}$, Ottavio Rena ${ }^{6,7}$, Chang Li $^{1,2}$, Jun Zhao ${ }^{1,2}$ \\ ${ }^{1}$ Department of Thoracic Surgery, The First Affiliated Hospital of Soochow University, Medical College of Soochow University, Suzhou, China; \\ ${ }^{2}$ Institute of Thoracic Surgery, The First Affiliated Hospital of Soochow University, Suzhou, China; ${ }^{3}$ Division of Thoracic and Endocrine Surgery, \\ University Hospitals of Geneva, Geneva, Switzerland; ${ }^{4}$ Department of MeSVA, University of L'Aquila, Thoracic Surgery Unit, "Giuseppe Mazzini”" \\ Hospital, Teramo, Italy; ${ }^{5}$ Thoracic Surgery and Lung Transplantation Unit, Fondazione IRCCS Ca' Granda Ospedale Maggiore Policlinico of \\ Milan, Milan, Italy; ${ }^{6}$ Thoracic Surgery, Ospedale Maggiore della Carità, Novara, Italy; ${ }^{7}$ Università del Piemonte Orientale, Novara, Italy \\ Contributions: (I) Conception and design: XY Zhu, C Ding, J Zhao, C Li; (II) Administrative support: J Zhao, C Li; (III) Provision of study materials \\ or patients: J Zhao, C Xu, C Ding, C Li; (IV) Collection and assembly of data: XY Zhu, C Ding; (V) Data analysis and interpretation: XY Zhu, C \\ Ding, C Li; (VI) Manuscript writing: All authors; (VII) Final approval of manuscript: All authors. \\ \#These authors contributed equally to this work. \\ Correspondence to: Chang Li; Jun Zhao. Department of Thoracic Surgery, The First Affiliated Hospital of Soochow University, Medical College of \\ Soochow University, Suzhou 215000, China. Email: cli@suda.edu.cn; zhaojia0327@126.com.
}

Background: Bronchial reconstruction is one of the most challenging procedures for thoracic surgeons. This study aimed to report the surgical techniques and clinical outcomes of bronchoplastic and sleeve resection for central lung cancer and summarize our center's experience of this challenging procedure over the past 8 years.

Methods: Between January 2013 and April 2021, 54 patients underwent a sleeve resection or a lobectomy with bronchoplasty, including 11 patients who received video-assisted thoracoscopic surgery (VATS) bronchial sleeve resection (4 via the uniportal approach and 7 via the biportal approach). Perioperative parameters and surgical short-term patient outcomes were analyzed to evaluate the safety and feasibility of this surgical procedure.

Results: The average operative time and blood loss were 247.8 73.1 (range, 126-455) minutes and $300.4 \pm 321.8$ (range, 50-1,500) $\mathrm{mL}$, respectively. The mean postoperative length of stay was $10.5 \pm 5.8$ (range, 4-29) days. Eleven patients underwent additional pulmonary angioplasty or sleeve resection. For patients who underwent biportal VATS sleeve lobectomy, the median operative time was 255 (interquartile range, 179-360) minutes, the median blood loss was 200 (interquartile range, 100-600) $\mathrm{mL}$, and the median postoperative hospital stay was 5 (interquartile range, 5-8) days. For patients who underwent uniportal VATS sleeve lobectomy, the median operative time was 288 (interquartile range, 241.5-343) minutes, the median blood loss was 75 (interquartile range, 50-100) $\mathrm{mL}$, and the median postoperative hospital stay was 5 (interquartile range, 4.5-5.5) days. No anastomosis-related complications or perioperative mortality was observed.

Conclusions: Both bronchoplastic resection and sleeve resection are safe and feasible procedures. Uniportal thoracoscopic sleeve lobectomy can be performed by skilled surgeons with satisfactory short-term outcomes, although it is surgically complicated.

Keywords: Bronchoplasty; sleeve lobectomy; lung cancer; video-assisted thoracic surgery (VATS); thoracotomy; uniportal video-assisted thoracic surgery (UVATS)

Submitted Sep 01, 2021. Accepted for publication Dec 08, 2021.

doi: $10.21037 /$ tlcr-21-913

View this article at: https://dx.doi.org/10.21037/tlcr-21-913 


\section{Introduction}

Complete resection, in order to achieve the oncological radicality, is the fundamental purpose of lung cancer treatment. In the past, pneumonectomy was a common choice for centrally located tumors involving the main stem bronchus or main artery, or extending into the origin of the lobar bronchus or artery. However, several publications in recent years revealed that bronchoplastic resection or sleeve lobectomy could be a valid alternative to pneumonectomy. This requires surgeons to complete airway reconstruction while removing the tumor. In fact, these procedures can not only achieve the same oncological outcome, but can also provide lower morbidity and mortality and improve postoperative quality of life by preserving pulmonary function (1-5).

Video-assisted thoracic surgery (VATS) was once considered as a contraindication for bronchoplasty and sleeve lobectomy due to the high risk of complications and the complexity of the operating steps, not to mention uniportal video-assisted thoracic surgery (UVATS). Owing to advancements in imaging systems and innovation of instrument designs, VATS procedures can be performed via the thoracoscopic approach to avoid pneumonectomy in selected patients without compromised lung function. Conventional VATS has been mostly conducted via three or four incisions. Uniportal thoracoscopic sleeve lobectomy was first reported in 2013 by Gonzalez-Rivas et al. (6). Subsequent studies described the more complex uniportal double sleeve resection and bronchoplastic and carinal sleeve procedures (7-11). In the hands of a skilled surgeon, thoracoscopic bronchial sleeve resection is an alternative and effective procedure for resectable central lung tumors, and can even be performed through a single incision. Rapid advances in minimally invasive techniques have also allowed surgeons to skip the multi-portal stage and directly perform biportal or even uniportal surgery in out institution (Figures 1,2).

This study reviewed the progress of bronchoplastic and sleeve resection in our institution over the last 8 years, concentrating on surgical outcomes of this challenging technique as well as the safety and feasibility of its application through VATS. We present the following article in accordance with the STROBE reporting checklist (available at https://dx.doi.org/10.21037/tlcr21-913).

\section{Methods}

\section{Patients}

We reviewed all cases of bronchoplastic resection or sleeve lobectomy of centrally located tumors invading the main stem bronchus or extending into the origin of the lobar bronchus performed in the Department of Thoracic Surgery of the First Affiliated Hospital of Soochow University (Suzhou, China) between January 2013 and April 2021. Before surgery, bronchoscopy was done to assess the tumor extension and determine the possibility for R0 resection. Enhanced chest computed tomography (CT), enhanced brain magnetic resonance imaging (MRI), wholebody bone scan, and abdominal and bilateral supraclavicular lymph node ultrasonography were routinely performed to exclude metastases. Positron emission tomography (PET)/ CT was used as an alternative for patients who could afford it. Since November 2019, our institution has applied preoperative three-dimensional CT bronchography and angiography (3D-CTBA) for the evaluation of pulmonary anatomical structure in patients undergoing lobectomy (Figures 1A,2A). Concrete steps of 3D reconstruction for pulmonary structure have been published previously $(12,13)$.

The indications for bronchoplastic resection or sleeve lobectomy were as follows: (I) centrally located lung tumor invading part of the main bronchus or lobar bronchus; (II) patient pulmonary function adequate to tolerate lobectomy. Systematic lymphadenectomy was conducted in all cases. Patients with distant metastasis were excluded.

This retrospective case series study was approved by the Ethics Committee of The First Affiliated Hospital of Soochow University (ethical approval No. 2021121). Individual consent was waived due to the retrospective nature of this study. Every procedure involving human participants in this study was performed in accordance with the Declaration of Helsinki (as revised in 2013). To protect personal privacy, patient data were handled anonymously.

\section{Surgical procedure}

Five thoracic surgeons, each of whom had completed at least 500 cases of pulmonary anatomical resection at our institution, conducted all the surgeries in this study. Most procedures performed during the study period were performed via thoracotomy. 

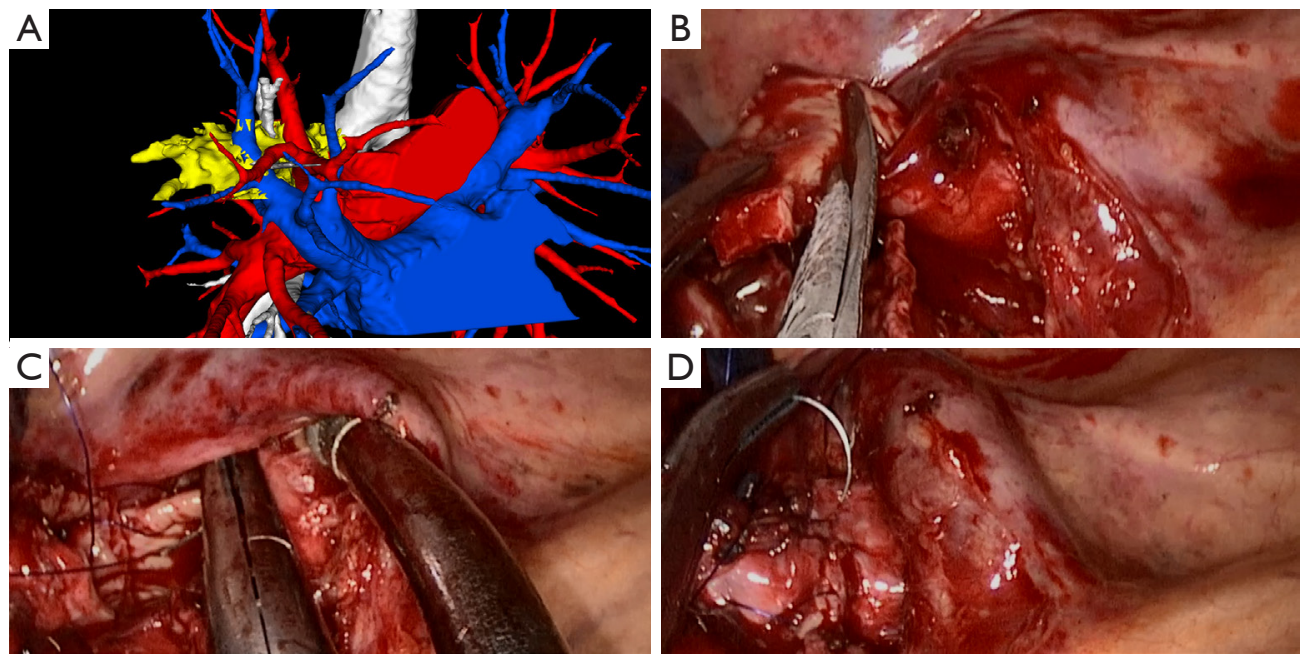

Figure 1 Uniportal video-assisted thoracoscopic right upper sleeve lobectomy in a patient. (A) Preoperative three-dimensional reconstruction of the right pulmonary anatomic structure conducted using Exoview. The bronchi are marked in white; the pulmonary arteries are marked in red; the pulmonary veins are marked in blue; the tumor is marked in yellow. (B) The distal bronchus was transected with scissors to adapt the diameter of the stump of the main bronchus. (C) A tensionless continuous suture was started from the posterior side. (D) The anterior wall of the bronchus was then sutured to complete the reconstruction.
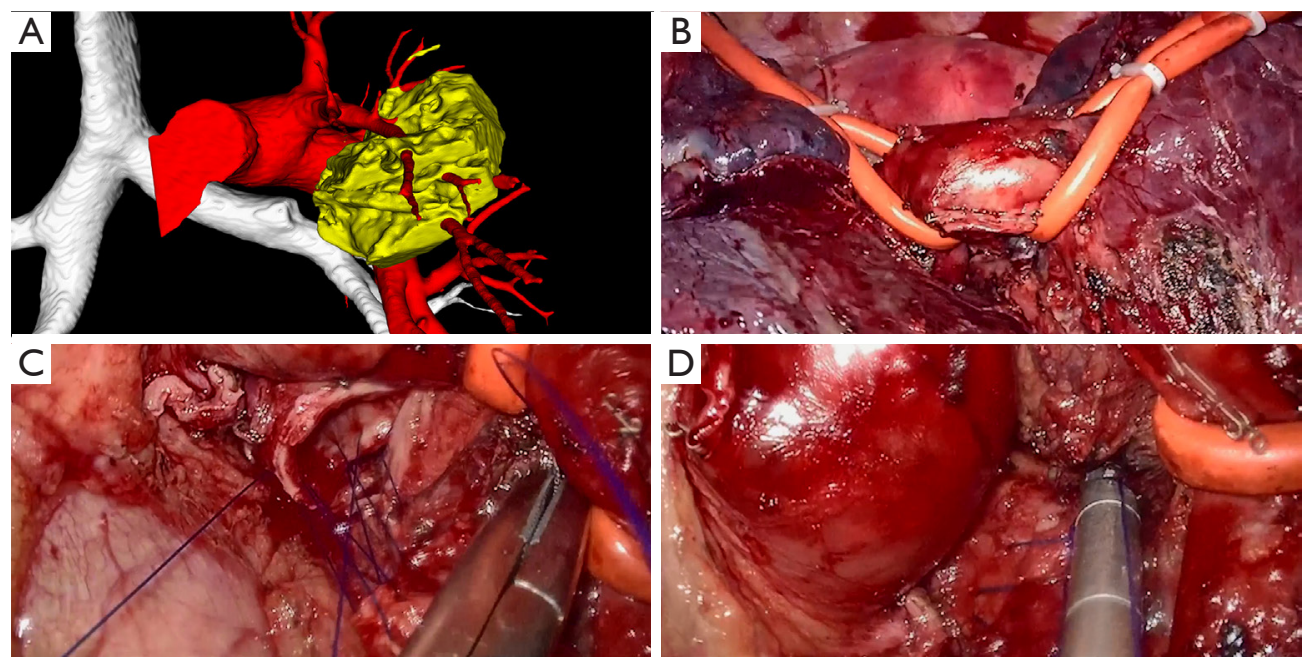

Figure 2 Uniportal video-assisted thoracoscopic left upper sleeve lobectomy in a patient. (A) Preoperative three-dimensional reconstruction of the left pulmonary anatomic structure conducted using Exoview. The bronchi are marked in white; the pulmonary arteries are marked in red; the tumor is marked in yellow. (B) Pulmonary artery suspension devices were used to reduce the complexity of the surgery. (C) A tensionless continuous suture was started from the posterior side. (D) The anterior side of the bronchus was then sutured to complete the reconstruction.

With the accumulation of sufficient VATS and conventional thoracotomy experience in our institution, we have applied biportal thoracoscopic sleeve lobectomy since December 2017, with the first uniportal thoracoscopic sleeve lobectomy being completed in December 2019. However, because of the high technical requirements, most sleeve lobectomy and bronchoplasty procedures are conducted via conventional thoracotomy, even when performed by the most 
experienced experts in our institution.

The patient was placed in the lateral decubitus position with the healthy side down, and single-lung ventilation general anesthesia was administrated. For conventional thoracotomy, an incision of $10-25 \mathrm{~cm}$ in length was made in the $4^{\text {th }}$ or $5^{\text {th }}$ intercostal space, depending on the tumor location and surgical preference of the surgeon. When necessary, a small part of the adjacent intercostal rib was removed to provide optimal exposure. For uniportal surgery, a single incision of $3-4 \mathrm{~cm}$ in length was made at the $4^{\text {th }}$ intercostal space along the anterior axillary line, without rib spreading. The incision was routinely covered with a silicon wound protector. With a 10-mm, 30-degree thoracoscope (Karl Storz, Tuttlingen, Germany) positioned at the superior side of the incision, other thoracoscopic instruments were concurrently attached to the single port beneath the thoracoscope. For biportal surgery, another $2-\mathrm{cm}$ assistant incision was made at the 8 th intercostal space on the posterior axillary line.

The bronchus was transected with a long handle scalpel and scissors (Figure 1B). To confirm negative resection margins, all specimens were sent for intraoperative frozen sectioning. If the bronchial margin was found to be positive for tumor cells, a 5 -mm wide margin would be cut from the positive side of the bronchial circumference each time. To provide more space for manipulation and save time, a systematic lymphadenectomy was routinely performed before the bronchoplasty or anastomosis. Further, the inferior pulmonary ligament was released to free the tension during anastomosis.

For right sleeve lobectomy, the azygos vein arch could be resected depending on surgical needs. For left sleeve lobectomy, pulmonary artery suspension devices could be applied to reduce the surgical complexity and improve the visual field and operating space, especially with the uniportal approach (Figure 2B). Afterward, as shown in Figure 1C,1D and Figure 2C,2D, bronchoplasty or end-to-end bronchial anastomosis was performed with continuous suturing using a single 3-0 prolene suture with a double needle. When performing a pulmonary angioplasty or double-sleeve resection, the surgeon would use a 4-0 prolene suture for vascular anastomosis. Then, a leakage test was performed to confirm no leakage under $30 \mathrm{cmH}_{2} \mathrm{O}$ airway pressure. Finally, after the completion of the procedure and depending on the surgical approach, one or two chest tubes were placed.

\section{Perioperative data collection}

The demographic features and perioperative results were reviewed for all cases. The clinical data collected included preoperative baseline features [age, sex, smoking history, drinking history, body mass index (BMI), maximum lesion diameter, lesion location, pulmonary function, preoperative complications, and whether neoadjuvant therapy was received], operative characteristics (surgical method, operative time, blood loss and stations, and the number of mediastinal lymph nodes dissected), routine pathological results, and short-term postoperative outcomes (duration and volume of chest drainage, postoperative hospital stay, and postoperative complications and mortality). Postoperative complications were graded according to the Clavien-Dindo classification (14). Minor complications requiring no treatment (i.e., grade I complications) were excluded. The patients' pathologic stages were assigned with reference to the $8^{\text {th }}$ TNM classification system. Perioperative mortality was defined as any patient death within 30 days after surgery. All the clinical data were collected separately by two investigators from patients' electronic records using HaiTai 3.0 software (Nanjing, Jiangsu, China). To reduce information bias, any conflicts between the two investigators were adjudicated by a third investigator. The sample size was determined by the number of patients undergoing such surgeries during the study period. All patients were followed up within 1 month after discharge by postoperative outpatient review or telephone.

\section{Statistical analysis}

Statistical analyses were performed using IBM SPSS Statistics (version 26.0, IBM Corp., Armonk, NY, USA). Continuous variables were expressed as the mean \pm standard deviation (SD) or as the median and range, while categorical data were presented as frequencies and percentages (\%).

\section{Results}

Between January 2013 and April 2021, 54 patients (50 men and 4 women) with a mean age of $61.2 \pm 9.3$ (range, 3680 ) years underwent a sleeve resection or a lobectomy with bronchoplasty. The clinical baseline characteristics of the patients are described in Table 1. Of the 54 patients, 4 had received neoadjuvant chemotherapy. The patients' tumor stages were classified according to the $8^{\text {th }}$ edition of the American Joint Committee on Cancer TNM classification system and are summarized in Table 2. The histological tumor diagnoses are presented in Table 3, with squamous cell carcinoma being the most frequent diagnosis $(n=43 / 54)$. 
Table 1 Patient clinical baseline characteristics

\begin{tabular}{|c|c|}
\hline Characteristics & Values \\
\hline Age, years & $61.2 \pm 9.3[36-80]$ \\
\hline \multicolumn{2}{|l|}{ Sex, n (\%) } \\
\hline Male & $50(92.6)$ \\
\hline Female & $4(7.4)$ \\
\hline $\mathrm{BMI}, \mathrm{kg} / \mathrm{m}^{2}$ & $23.2 \pm 3(18-30.1)$ \\
\hline Smoking history, n (\%) & $43(79.6)$ \\
\hline Drinking history, n (\%) & $14(25.9)$ \\
\hline Neoadjuvant therapy, n (\%) & $4(7.4)$ \\
\hline FEV1, L & $2.3 \pm 0.6(1.14-3.75)$ \\
\hline$\% F E V 1, \%$ & $74.1 \pm 14.5(37.6-102.8)$ \\
\hline$\% \mathrm{MVV}, \%$ & $71.9 \pm 17.5(35.6-112.5)$ \\
\hline Hypertension, n (\%) & $13(24.1)$ \\
\hline Diabetes mellitus, $\mathrm{n}(\%)$ & $3(5.5)$ \\
\hline Maximal lesion size, $\mathrm{cm}$ & $3.6 \pm 1.7(1.2-10)$ \\
\hline \multicolumn{2}{|l|}{ Lesion location, n (\%) } \\
\hline Left upper lobe & $18(33.3)$ \\
\hline Right upper lobe & $31(57.4)$ \\
\hline Left lower lobe & $1(1.9)$ \\
\hline Left lower lobe and lingular division & $1(1.9)$ \\
\hline Right middle and lower lobe & $2(3.7)$ \\
\hline Tracheal carina & $1(1.9)$ \\
\hline
\end{tabular}

BMI, body mass index; FEV1, forced expiratory volume in 1 $\mathrm{s}$; \%FEV1, percentile forced expiratory volume in 1 second; $\% \mathrm{MVV}$, percentile maximal voluntary ventilation.

Table 2 Stage classification

\begin{tabular}{lc}
\hline Stage & No. of cases \\
\hline IB & 17 \\
IIA & 2 \\
IIB & 20 \\
IIIA & 12 \\
IIIB & 3 \\
\hline
\end{tabular}

All patients were offered an R0 resection. The patients' surgical outcomes are shown in Table 4. The mean operative time and mean blood loss were 247.8 \pm 73.1 (range, 126455 ) minutes and $300.4 \pm 321.8$ (range, 50-1,500) $\mathrm{mL}$,
Table 3 Histological type of surgically removed specimens

\begin{tabular}{lc}
\hline Histological type & No. \\
\hline Squamous cell carcinoma & 43 \\
Adenocarcinoma & 4 \\
Atypical carcinoid & 1 \\
Mucoepidermoid carcinoma & 2 \\
Adenosquamous carcinoma & 1 \\
Colorectal carcinoma metastasis & 1 \\
Small cell carcinoma & 1 \\
Adenoid cystic carcinoma & 1 \\
Total & 54 \\
\hline
\end{tabular}

respectively. The mean chest tube duration was $8.4 \pm 5$ (range, 2-26) days, and the mean postoperative length of stay was $10.5 \pm 5.8$ (range, 4-29) days.

Due to the local extent of tumor invasion, some patients underwent additional surgical procedures, which are detailed in Table 5. Eleven patients underwent an additional pulmonary angioplasty or sleeve resection. In one case, superior vena cava replacement was conducted due to palpation of the tumor thrombus in the superior vena cava during the operation. In another case, part of the pericardium and the left atrium was removed due to tumor invasion. Systematic lymph node dissection was conducted after lesion resection, and malignancy was confirmed by frozen section examination. The average numbers of mediastinal lymph node stations and lymph nodes dissected were $3.7 \pm 1.2$ (range, $1-7$ ) and $13.1 \pm 7.9$ (range, 2-37), respectively.

Bacterial pneumonia was the most common postoperative complication $(n=25 / 54)$, followed by prolonged ( $>7$ days) air leakage $(n=18 / 54)$. All affected patients were managed conservatively through adjusting antibiotic use and replacing the negative pressure drainage bottle. Severe atelectasis occurred in 4 patients $(7.4 \%), 3$ of whom underwent fiberoptic bronchoscopy for efficient aspiration of the secretions, and 1 had a tracheotomy. One patient (1.9\%) experienced postoperative bleeding following a right upper sleeve lobectomy, from the mediastinal pleura in front of the superior vena cava. Consequently, the patient underwent unplanned reoperation on day 16 after the first operation. One patient $(1.9 \%)$ developed paroxysmal atrial fibrillation postoperatively, and sinus rhythm was restored after amiodarone administration. Another patient (1.9\%) 
Table 4 Patient surgical outcomes

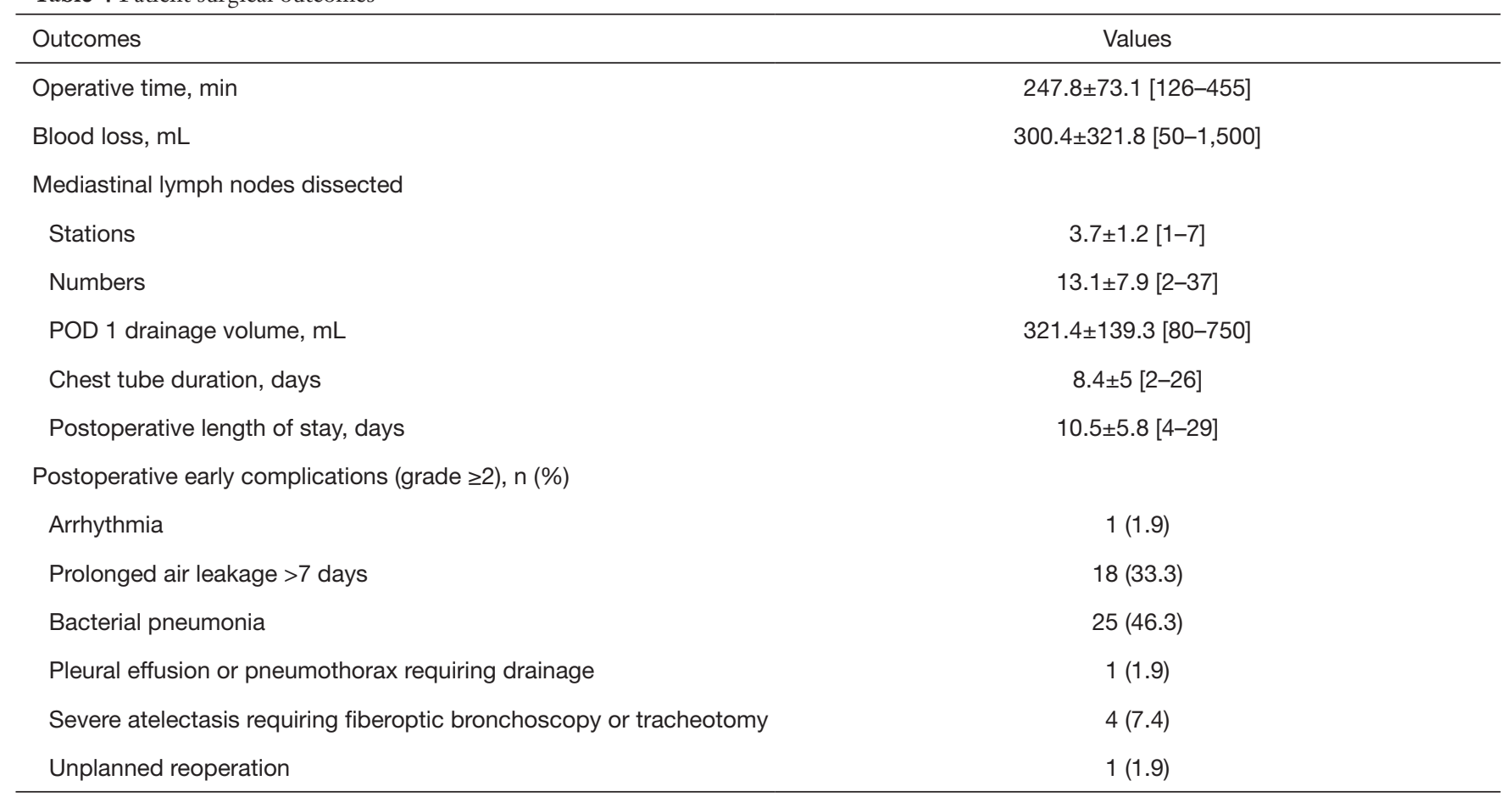

POD 1, postoperative day 1.

Table 5 Distribution per type of bronchoplasty and sleeve resection

\begin{tabular}{|c|c|}
\hline Surgical method & No. \\
\hline Sleeve lobectomy & 32 \\
\hline Double-sleeve lobectomy & 5 \\
\hline Half-carinal sleeve resection & 3 \\
\hline Bronchoplasty with pulmonary angioplasty or vascular sleeve resection & 2 \\
\hline Sleeve lobectomy with pulmonary angioplasty & 4 \\
\hline Sleeve lobectomy with replacement of superior vena cava & 1 \\
\hline Sleeve lobectomy with pericardial and atrial resection & 1 \\
\hline
\end{tabular}

received closed thoracic drainage on days 7 and 8 after surgery, due to pleural effusion and pneumothorax. There were no cases of readmission or 30-day mortality.

Eleven VATS sleeve lobectomies (4 via the uniportal approach and 7 via the biportal approach) were completed uneventfully without conversion to conventional thoracotomy. The detailed clinical data of these cases are listed in
Tables 6,7. No perioperative mortality was reported. The first case was performed using a conventional twoport approach in December 2017. The median operative time was 255 (interquartile range, 179-360) minutes, and the median blood loss was 200 (interquartile range, 100-600) $\mathrm{mL}$. The median number of mediastinal lymph nodes dissected was 8 (interquartile range, $3-12$ ), and the 
Table 6 Perioperative parameters of patients who underwent biportal video-assisted thoracoscopic sleeve lobectomy

\begin{tabular}{|c|c|}
\hline Parameters & Values \\
\hline Median age, years, [IQR] & 57 [52-62] \\
\hline \multicolumn{2}{|l|}{ Sex, n (\%) } \\
\hline Male & $5(71.4)$ \\
\hline Female & $2(28.6)$ \\
\hline Neoadjuvant therapy, n (\%) & 0 \\
\hline Median maximal lesion size, cm, [IQR] & $3[1.5-4]$ \\
\hline \multicolumn{2}{|l|}{ Lesion location, n (\%) } \\
\hline Left upper lobe & $3(42.9)$ \\
\hline Right upper lobe & $4(57.1)$ \\
\hline \multicolumn{2}{|l|}{ Histological type, n (\%) } \\
\hline Squamous cell carcinoma & $4(57.1)$ \\
\hline Adenocarcinoma & $1(14.3)$ \\
\hline Atypical carcinoid & $1(14.3)$ \\
\hline Mucoepidermoid carcinoma & $1(14.3)$ \\
\hline Median operative time, min, [IQR] & 255 [179-360] \\
\hline Median blood loss, mL, [IQR] & $200[100-600]$ \\
\hline \multicolumn{2}{|l|}{ Median mediastinal lymph nodes dissected, $\mathrm{n}$ [IQR] } \\
\hline Stations & $3[3-4]$ \\
\hline Numbers & 8 [3-12] \\
\hline \multicolumn{2}{|l|}{ TNM stage, n (\%) } \\
\hline IB & $3(42.9)$ \\
\hline IIB & $3(42.9)$ \\
\hline IIIA & $1(14.3)$ \\
\hline Median POD 1 drainage volume, ml, [IQR] & $370[250-400]$ \\
\hline Median chest tube duration, days, [IQR] & $4[3-6]$ \\
\hline Median postoperative length of stay, days, [IQR] & $5[5-8]$ \\
\hline \multicolumn{2}{|l|}{ Postoperative early complications (grade $\geq 2$ ), n (\%) } \\
\hline Prolonged air leakage $>7$ days & $1(14.3)$ \\
\hline Bacterial pneumonia & $1(14.3)$ \\
\hline
\end{tabular}

POD 1, postoperative day 1 IQR, interquartile range.

median postoperative hospital stay was 5 (interquartile range, 5-8) days. One patient developed pulmonary infections after surgery but recovered uneventfully after their antibiotic use was adjusted. Another patient suffered prolonged air leakage and was treated with conservative
Table 7 Perioperative parameters of patients who underwent uniportal video-assisted thoracoscopic sleeve lobectomy

\begin{tabular}{|c|c|}
\hline Parameters & Values \\
\hline Median age, years, [IQR] & $63.5[56.5-70.5]$ \\
\hline \multicolumn{2}{|l|}{ Sex, n (\%) } \\
\hline Male & $4(100.0)$ \\
\hline Female & 0 \\
\hline Neoadjuvant therapy, n (\%) & $1(25.0)$ \\
\hline Median maximal lesion size, cm, [IQR] & $2.5[2.1-3.75]$ \\
\hline \multicolumn{2}{|l|}{ Lesion location, n (\%) } \\
\hline Left upper lobe & $1(25.0)$ \\
\hline Right upper lobe & $3(75.0)$ \\
\hline \multicolumn{2}{|l|}{ Histological type, n (\%) } \\
\hline Squamous cell carcinoma & $4(100.0)$ \\
\hline Median operating time, min, [IQR] & 288 [241.5-343] \\
\hline Median blood loss, mL, [IQR] & $75[50-100]$ \\
\hline \multicolumn{2}{|l|}{ Median mediastinal lymph nodes dissected, $\mathrm{n}$ [IQR] } \\
\hline Stations & 4 [3-5] \\
\hline Numbers & 13 [6.5-18] \\
\hline \multicolumn{2}{|l|}{ TNM stage, n (\%) } \\
\hline IB & $1(25.0)$ \\
\hline IIB & $3(75.0)$ \\
\hline Median POD 1 drainage volume, mL, [IQR] & $100[90-160]$ \\
\hline Median chest tube duration, days, [IQR] & $4[3-4.5]$ \\
\hline Median postoperative length of stay, days, [IQR] & $5[4.5-5.5]$ \\
\hline \multicolumn{2}{|l|}{ Postoperative early complications (grade $\geq 2$ ), n (\%) } \\
\hline Bacterial pneumonia & $1(25.0)$ \\
\hline
\end{tabular}

POD 1, postoperative day 1 ; IQR, interquartile range.

therapy for 20 days. Pathological findings of the 7 cases in which the biportal approach was used included 4 cases of squamous cell carcinoma, 1 case of adenocarcinoma, 1 case of mucoepidermoid carcinoma, and 1 case of atypical carcinoid. TNM staging of non-small cell lung cancer revealed 3 cases with stage IB, 3 patients with stage IIB, and 1 patient with stage IIIA. After December 2019, another 4 uniportal surgeries mentioned above were conducted successively. One of the patients received 2 cycles of neoadjuvant chemotherapy (docetaxel + carboplatin). The median operative time was 288 (interquartile range, 
241.5-343) minutes, and the median blood loss was 75 (interquartile range, $50-100) \mathrm{mL}$. The median number of mediastinal lymph nodes dissected was 13 (interquartile range, 6.5-18), and the median postoperative hospital stay was 5 (interquartile range, 4.5-5.5) days. One patient suffered pulmonary infection after surgery but recovered uneventfully after antibiotic escalation. All the cases were diagnosed as squamous cell carcinoma. TNM staging of non-small cell lung cancer revealed 1 case of IB and 3 cases of IIB.

\section{Discussion}

The first bronchial sleeve resection was performed by Thomas for a patient with a right main bronchus tumor in 1956 (15). Since then, multiple publications have shown that sleeve lobectomy reduces lung function loss and improves surgical mortality and survival rates compared with pneumonectomy $(1-5,16)$. Additionally, sparing lung parenchyma can undoubtedly improve the quality of life of patients after surgery due to them having a greater pulmonary reserve, which has been advocated as one of the most reliable indicators influencing the decision to perform a sleeve lobectomy. Therefore, an increasing number of thoracic surgeons prefer sleeve resection as an alternative to pneumonectomy for patients with central lung cancer. Despite the unquestionable benefits of bronchial reconstruction in preventing pneumonectomy, most of these procedures are still performed by thoracotomy in our institution due to their complexity.

At one time, VATS was considered to be a technically restricted area for sleeve lobectomy and bronchoplasty. Major concerns have surrounded the completeness of oncologic resection and the safety of anastomosis. Due to technical difficulties in anatomical reconstruction and airway management, the use of a thoracoscopic approach for these procedures represents a challenge, even for experienced thoracic surgeons. Nevertheless, minimally invasive technologies have developed rapidly over the past 20 years, and the advent of UVATS has accelerated this evolution. In 2002, the development of new instruments and experience accumulated from conventional thoracoscopic lobectomies made this method possible by Santambrogio et al. (17). After sufficient experience gained with biportal and uniportal VATS, authors first performed the VATS sleeve bronchoplasty for a patient with mucoepidermoid carcinoma of the left lower lobar bronchus in 2017. Interestingly, conventional VATS has been mostly performed with three or four incisions. Owing to rapid development of surgical instruments and techniques, multi-portal VATS may not be a necessary learning stage for bronchoplastic or sleeve resection.

Although UVATS provides direct vision and is a similar surgical approach to thoracotomy, it entails a restricted visual field and causes interference between surgical instruments. End-to-end anastomosis of the bronchus is an extremely challenging procedure using a UVATS approach. To our knowledge, in China, only a few experienced surgical teams in high-volume centers have successfully adopted UVATS for bronchial sleeve resection $(9,11,18,19)$ since it was first reported by Gonzalez-Rivas et al. in 2013 (6). Zhao et al. (11) highlighted that UVATS shows a trend toward a reduction of patients' postoperative pain and shorter postoperative hospital stay, also helping to alleviate the corresponding financial burden. Another advantage of UVATS is its superior cosmetic results, which is, to some extent, important for young patients. In December 2017, our unit conducted the first biportal VATS left upper sleeve lobectomy, and since December 2019, 4 successive UVATS sleeve lobectomies have been performed uneventfully without conversion to thoracotomy. All the uniportal surgeries were completed by the same surgeon (J. Zhao). Vascular and carinal reconstructions are known to be more difficult than bronchial reconstruction, although GonzalezRivas successfully completed these procedures using a single port approach $(20,21)$. Given the complexity of the procedure and our lack of surgical experience, we have yet to apply the uniportal technique in complex sleeve resection such as double-sleeve lobectomy, half-carinal, and carinal sleeve resection.

In order to conduct VATS sleeve lobectomy successfully, we summarize our experience from three aspects. First, the proper patient selection and an adequate preoperative evaluation. Patients with large central tumors should undergo careful assessment, including the relationship between the tumor, hilar vessels, and lymph nodes. Enhanced CT scans and bronchoscopy are also necessary, and three-dimensional reconstruction, which can provide a more stereo and concrete observation of the pulmonary anatomical structures, is recommended if possible. Second, our research revealed that it is more difficult to carry out the reconstruction procedures after neoadjuvant therapy not only due to the narrowing of the space between vessels and the bronchus, but also for the increase in vascular fragility and toughness of the mediastinal lymph nodes. These considerations are in agreement with the experience of Liang et al. (22). Moreover, sufficient exposure of the operating space is also crucial, and the field of vision offered 
by a single incision is limited. This disadvantage becomes even more apparent during left upper sleeve lobectomy due to the obstruction of the aortic arch. Therefore, based on Huang's report (19), we used a set of pulmonary artery suspension devices to expose the bronchus and greatly reduce the interference from the pulmonary artery, thus preventing injury to the anastomotic stoma. In addition, preferential dissection of mediastinal lymph nodes can provide an excellent field of view when conducting anastomosis. Third, considering that sleeve resection is a particularly complex procedure, there is no convincing evidence favoring interrupted or continuous sutures for bronchial anastomosis at present. Based on our limited experience, we recommend complete continuous suture to enable a tension-free anastomosis, which seems to be safe and with no anastomosis-related complications, such as bronchopleural fistula or anastomotic stricture. A double needle with 3-0 prolene sutures should be used to avoid intertwining. Further, according to Zhou's study (23), knots placed in the bronchial lumen may cause irritable cough, retention of secretions, or even anastomotic stricture. A continuous seam ensures better visibility and more manipulation space for the surgeon. Meanwhile, releasing the inferior pulmonary ligament and freeing the bronchus for as long as possible can ensure a tension-free anastomosis. In addition to allowing for easier manipulation, these techniques could also greatly reduce the operative time.

The major concern with sleeve lobectomy and bronchoplasty relates to anastomosis-related complications, such as bronchopleural fistula or anastomotic stricture. Tapias et al. (24) reported an incidence rate to $0-8.1 \%$ of bronchopleural fistula in patients undergoing sleeve resection. A meta-analysis published in 2012 observed that postoperative morbidity and mortality hinge on the healing of the anastomosis (25). However, no such complications were observed in our cohort. In our institution, intra- and postoperative bronchoscopy was not carried out as a routine surveillance procedure without strong clinical indication such as unsatisfactory intraoperative anastomosis procedure, severe atelectasis or high suspicion of complications associated with anastomosis. Pulmonary infection and air leakage were two of the most common complications in our series. All the patients with these complications were treated conservatively by upgrading antibiotics or applying a negative pressure drainage system. However, the incidence of pneumonia is very high (near 50\%). A possible cause is different evaluation criteria. We adjust treatment plan mainly according to blood routine test or imaging results such as bedside chest radiography instead of strict bacteriological examination. Interestingly, there was an incidence of $2 / 11$ cases (near 20\%) of bacterial pneumonia during the postoperative period among patients treated through VATS, which is lower than the one treated through thoracotomy. However, we did not perform statistical comparison between groups due to the sample size.

This retrospective study has some limitations. First, because all the patients were treated at a single center, the sample size was small. Second, the investigators had to rely on old patient e-files, which are not always complete and precise, and may have led to errors. Third, no long-term follow-up data, such as 5-year disease-free survival or overall survival, were analyzed in our study, as we could not obtain accurate information regarding loco-regional recurrence or long-term survival. In future, a high-volume prospective randomized controlled trial needs to be conducted to confirm the advantages of UVATS on perioperative and long-term outcomes.

\section{Conclusions}

In conclusion, both bronchoplastic resection and sleeve resection are safe and feasible procedures. Although this is a small retrospective case series, our experience shows that uniportal thoracoscopic sleeve lobectomy can be performed by skilled surgeons with satisfactory short-term outcomes, although it is surgically complicated.

\section{Acknowledgments}

The authors appreciate the academic support from the AME Thoracic Surgery Collaborative Group.

Funding: This study was funded by the Key Clinical Special Program of Suzhou Government (grant No. LCZX201902).

\section{Footnote}

Reporting Checklist: The authors have completed the STROBE reporting checklist. Available at https://dx.doi. org/10.21037/tlcr-21-913

Data Sharing Statement: Available at https://dx.doi. org/10.21037/tlcr-21-913

Conflicts of Interest: All authors have completed the ICMJE uniform disclosure form (available at https://dx.doi. org/10.21037/tlcr-21-913). The authors have no conflicts of 
interest to declare.

Ethical Statement: The authors are accountable for all aspects of the work in ensuring that questions related to the accuracy or integrity of any part of the work are appropriately investigated and resolved. This retrospective case series study was approved by the Ethics Committee of The First Affiliated Hospital of Soochow University (ethical approval No. 2021121). Individual consent was waived due to the retrospective nature of this study. Every procedure involving human participants in this study was performed in accordance with the Declaration of Helsinki (as revised in 2013).

Open Access Statement: This is an Open Access article distributed in accordance with the Creative Commons Attribution-NonCommercial-NoDerivs 4.0 International License (CC BY-NC-ND 4.0), which permits the noncommercial replication and distribution of the article with the strict proviso that no changes or edits are made and the original work is properly cited (including links to both the formal publication through the relevant DOI and the license). See: https://creativecommons.org/licenses/by-nc-nd/4.0/.

\section{References}

1. Ma Z, Dong A, Fan J, et al. Does sleeve lobectomy concomitant with or without pulmonary artery reconstruction (double sleeve) have favorable results for non-small cell lung cancer compared with pneumonectomy? A meta-analysis. Eur J Cardiothorac Surg 2007;32:20-8.

2. Okada M, Yamagishi H, Satake S, et al. Survival related to lymph node involvement in lung cancer after sleeve lobectomy compared with pneumonectomy. J Thorac Cardiovasc Surg 2000;119:814-9.

3. Li Z, Chen W, Xia M, et al. Sleeve lobectomy compared with pneumonectomy for opera-ble centrally located non-small cell lung cancer: a meta-analysis. Transl Lung Cancer Res 2019;8:775-86.

4. Schirren J, Bölükbas S, Bergmann T, et al. Prospective study on perioperative risks and functional results in bronchial and bronchovascular sleeve resections. Thorac Cardiovasc Surg 2009;57:35-41.

5. Yildizeli B, Fadel E, Mussot S, et al. Morbidity, mortality, and long-term survival after sleeve lobectomy for non-small cell lung cancer. Eur J Cardiothorac Surg 2007;31:95-102.

6. Gonzalez-Rivas D, Fernandez R, Fieira E, et al. Uniportal video-assisted thoracoscopic bronchial sleeve lobectomy: first report. J Thorac Cardiovasc Surg 2013;145:1676-7.

7. Gonzalez-Rivas D, Delgado M, Fieira E, et al. Double sleeve uniportal video-assisted thoracoscopic lobectomy for non-small cell lung cancer. Ann Cardiothorac Surg 2014;3:E2.

8. Dai L, Jiang L, Gu Y, et al. Modified double lumen tube for a unique bronchial and cari-nal resection in a patient undergoing uniportal VATS for tumour: A case report. Transl Cancer Res 2020;9:2077-81.

9. Huang $\mathrm{L}$, Zheng J, Hu R, et al. Uniportal video-assisted thoracoscopic surgery for lung neoplasms with tracheal bronchus: a case report. Transl Cancer Res 2020;9:3721-4.

10. Ma S, Yan T, Liu D, et al. Initial experience of sleeve lobectomy under complete video-assisted thoracic surgery. Asia Pac J Clin Oncol 2018;14:114-9.

11. Zhao J, Zeng Q, Li J, et al. Uniportal versus multiportal thoracoscopic sleeve lobectomy for the surgical treatment of centrally located lung cancer: a single institution experience. J Thorac Dis 2020;12:7145-55.

12. Zhu XY, Yao FR, Xu C, et al. Utility of preoperative three-dimensional CT bronchography and angiography in uniportal video-assisted thoracoscopic anatomical lobectomy: a retrospective propensity score-matched analysis. Ann Transl Med 2021;9:480.

13. Cui Z, Ding C, Li C, et al. Preoperative evaluation of the segmental artery by three-dimensional image reconstruction vs. thin-section multi-detector computed tomography. J Thorac Dis 2020;12:4196-204.

14. Dindo D, Demartines N, Clavien PA. Classification of surgical complications: a new proposal with evaluation in a cohort of 6336 patients and results of a survey. Ann Surg 2004;240:205-13.

15. THOMAS CP. Conservative resection of the bronchial tree. J R Coll Surg Edinb 1956;1:169-86.

16. Burfeind WR Jr, D'Amico TA, Toloza EM, et al. Low morbidity and mortality for bronchoplastic procedures with and without induction therapy. Ann Thorac Surg 2005;80:418-21; discussion 422.

17. Santambrogio L, Cioffi U, De Simone M, et al. Videoassisted sleeve lobectomy for mucoepidermoid carcinoma of the left lower lobar bronchus: a case report. Chest 2002;121:635-6.

18. Soultanis KM, Chen Chao M, Chen J, et al. Technique and outcomes of 79 consecutive uniportal videoassisted sleeve lobectomies. Eur J Cardiothorac Surg 2019;56:876-82.

19. Huang L, Wu P, Zhao L, et al. Reduced complexity of 
uniportal video-assisted thoracoscopic left upper sleeve lobectomy. J Thorac Dis 2018;10:3791-6.

20. Gonzalez-Rivas D, Garcia A, Chen C, et al. Technical aspects of uniportal video-assisted thoracoscopic double sleeve bronchovascular resections. Eur J Cardiothorac Surg 2020;58:114-22.

21. Gonzalez-Rivas D, Yang Y, Stupnik T, et al. Uniportal video-assisted thoracoscopic bronchovascular, tracheal and carinal sleeve resections $†$. Eur J Cardiothorac Surg 2016;49 Suppl 1:i6-16.

22. Liang H, Yang C, Gonzalez-Rivas D, et al. Sleeve lobectomy after neoadjuvant chemoimmunotherapy/ chemotherapy for local advanced non-small cell lung

Cite this article as: Zhu XY, Ding C, Xu C, Chen J, Ju S, Pan S, Cui ZH, Bédat B, Divisi D, Tosi D, Rena O, Li C, Zhao J. Techniques and outcomes of bronchoplastic and sleeve resection: an 8-year single-center experience. Transl Lung Cancer Res 2021;10(12):4538-4548. doi: 10.21037/tlcr-21-913 cancer. Transl Lung Cancer Res 2021;10:143-55.

23. Zhou S, Pei G, Han Y, et al. Sleeve lobectomy by videoassisted thoracic surgery versus thoracotomy for non-small cell lung cancer. J Cardiothorac Surg 2015;10:116.

24. Tapias LF, Ott HC, Mathisen DJ. Complications Following Carinal Resections and Sleeve Resections. Thorac Surg Clin 2015;25:435-47.

25. Shi $W$, Zhang $W$, Sun $H$, et al. Sleeve lobectomy versus pneumonectomy for non-small cell lung cancer: a metaanalysis. World J Surg Oncol 2012;10:265.

(English Language Editor: J. Reylonds) 